\title{
EL SUPERLATIVO EN LA BIBLIA LATINA
}

A study on the superlative of the Latin Bible, specially the Vulgata, trying to track back the possible influences of Hebrew and Greek.

Como es sabido, el superlativo latino puede ser absoluto y relativo. $\mathrm{E} 1 \mathrm{a} \mathrm{b}$ s o $1 \mathrm{u}$ t o tiene valor intensivo. Indica que el ser posee la cualidad de que se trata en un grado elevado: altissimus 'altísimo', 'muy alto'. El re la ti vo indica que el ser posee la cualidad en el grado más alto de entre tres o más componentes de un grupo: altissimus omnium, 'el más alto de todos'.

La diferencia entre el comparativo y superlativo radica únicamente en que en el comparativo la comparación se establece entre dos objetos o conceptos; en el superlativo, en cambio, la comparación se hace entre tres o más.

Dada esta estrecha relación entre el comparativo y el superlativo, 1a gradación del adjetivo no siempre se mantiene dentro de sus propios límites y por eso pueden darse y de hecho se dan numerosas interferencias. A continuación veremos muchos casos de estas interferencias en el latín biblico.

En español, como en las demás lenguas románicas, tanto el comparativo como el superlativo orgánicos - fuera de algunos pocos casosfueron sustituidos por perffrasis analíticas: más... que para el comparativo de superioridad; menos... que para el comparativo de inferioridad; tan... como para el comparativo de igualdad, o por los adverbios más y menos, precedidos del artículo (el más, el menos) y seguidos de la preposición de para expresar el único superlativo español que atribuye a un objeto el grado máximo de la cualidad entre los demás objetos con que se le compara, por ejemplo, el más pequeño de la casa, el menos conocido de todos, etc. Los pocos superlativos orgánicos heredados del latín significan, o el grado más alto de una cualidad (tiempo máximo), 
o una intensificación de la misma, equivalente a la que se expresa con el adverbio muy (muy malo) o el sufijo -ísimo (malísimo) ${ }^{1}$.

Para exponer correctamente, y entender, el superlativo en la Biblia latina hay que partir necesariamente de la lengua hebrea y de sus formas de expresiónl del superlativo, pues la Biblia latina es en última instancia una traducción de un original hebreo (y arameo) y griego, pero cuando se trata de la traducción de textos griegos, este mismo original griego está influido por la mentalidad y la lengua hebrea (o aramea) de sus autores.

Pues bien, en hebreo ( $\mathrm{y}$ arameo) existen también, como en latín, dos tipos de superlativo, el relativo y el absoluto. E1 hebreo (y el arameo) carecen de superlativos orgánicos ${ }^{2}$, y por eso, tanto el comparativo como el superlativo se expresan siempre por medio de adjetivos (o nombres) en grado positivo.

\section{SUPERLATIVO RELATIVO.}

En hebreo puede expresarse de varias maneras:

\section{A) Adjetivo determinado por el artículo.}

Este tipo de superlativo presenta en la Biblia latina las siguientes posibilidades:

I. Superlativo:

I. Dauid autem erat minimus (de entre varios hermanos) (I Sam. I7, I4.

2. Et oliueta optima (de todos) tollet (I Sam. 8, I4).

3. Populum istum pessimum (de todos) (Ier. 13,Io).

4. Pulcherrima inter mulieres (Cant. I, 7).

2. Comparativo por superlativo:

I. Vos plures (= plurimi) estis (I Re. I8, 25).

2. Eligite meliorem (=optimum) (2 Re. I0, 3).

3. Filius suus minor (= minimus) (Gen. 9, 24). p. 226.

1 Cf. S. Gill Gaya, Curso superior de sintaxis española, Barcelona, 1961,

2 Cf. P. Joüon, Grammaire de l'hébreu biblique, Roma, 1947,, p. 437 s.; H. BauerP. Leander, Grammatik des Biblisch-Aramäischen, Hildesheim (reimpr.), 1962, p. 3198 . 
3. Positivo por superlativo:

I. A paruo (= minimo) usque ad magnum (= maximum) (I Sam. 30,19).

2. Superbiam Ierusalem multam (= plurimam) (Ier. I3,9).

3. Homo delicatus (= delicatissimus) in te (Deut. 28,54).

4. Nomen magnorum (= maximorum) qui sunt in terra (2 Sam. $7,9)$.

5. Benedicta (= benedictissima) tu inter mulieres (Luc. I,42).

B) Adjetivo determinado por un nombre determinado.

Este tipo de superlativo hebreo ofrece también en la Biblia latina varias posibilidades:

I. Superlativo:

I. De minima tribu Israel (I Sam. 9,2I).

2. Quatuor sunt minima terrae (Prou. 30,24).

3. Ioachaz, minimus (filiorum eius) (2 Cro. 21,17 ).

4. Sacerdos maximus inter fratres suos (Leu. $2 \mathrm{I}, \mathrm{IO})$.

5. Optimis gregibus ouium (I Sam. 15,9).

6. Pessimos de gentibus (Ez. 7,24).

2. Comparativo por superlativo:

I. Pepercit populus melioribus ouibus (= optimis ouium (I Sam.) I5, I5).

2. Maiores natu Israel (= maximi Israel) (I Re. 8, I); cf. 2 Sam. 17,4).

3. Maiores natu Iuda (= maximi Iuda) (2 Sam. I9, II); cf. 2 Cro. $34,29)$.

3. Positivo por superlativo:

I. Sacerdos magnus (= maximus) e fratribus suis (Leu. 2I, IO VL).

2. Bellatorum fortium (= fortissimorum) de filiis Cedar (Is. 2I,I7).

3. Robustus (= robustissimus) corde inter fortes (Am. 2,16).

4. Omnes fortes (= fortissimi) manu (Ios. I,I4).

5. Dominus magnus (= maximus) fortitudine (Nah. I,3). 
C) Adjetivo determinado por un sufijo.

Este tipo de superlativo hebreo presenta igualmente en la Biblia latina varias traducciones:

I. Superlativo:

I. Qui optimus in eis est (Miq. 7,4).

2. Fortissimus eorum (I Sam. I7,5I).

3. A minimo eorum usque ad maximum (eorum) $(\text { Ier. } 3 \mathrm{I}, 34)^{1}$.

2. Comparativo por superlativo:

I. A maiore (eorum) (= maximo) usque ad minorem (eorum) (= minimum) (Ion. 3,5).

2. A minore (eorum) (= minimo) usque ad maioren (eorum) (= maximum) (Ier. 6,13).

3. Maiores (eorum) (= maximi) miserunt minores suos (= minimos) ad aquam (Ier. I4,3).

3. Positivo por superlativo:

I. Vocaui fortes (= fortissimos) meos in ira mea (Is. 13,3).

2. Gladius ad fortes (= fortissimos) illius (Ier. 50,36).

3. Et apprehensi sunt fortes (= fortissimi) eius (Ier. 5I,56).

Además de estas interferencias entre los distintos grados del adjetivo, limitadas a unos cuantos ejemplos de cada tipo, tomadas sólo de los libros traducidos del hebreo, existen otras, que vamos a mencionar con algunos ejemplos.

\section{D) Comparativo por positivo.}

I. Melius (= bonum) est consilium Chusai (2 Sam. I7, I4).

2. Videbis abominationes maiores (= magnas) (Ez. VIII, 6,13). En $E z$. VIII, 6, la Vulgata traduce otra vez correctamente el texto diciendo: abominationes magnas.

1 El texto paralelo de Hebr. 8, i I traduce con un comparativo un positivo griego: a minore usque ad maiorem eorum. La confusión entre los grados del adjetivo es evidente. Véanse otros ejemplos de superlativos por positivos y de superlativos por comparativos en $\mathrm{H}$. Rönsch, Itala und Vulgata, Munich (reimpr.), 1965, p. 4I 5 ss.; F. Kaulen, Sprachliches Handbuch zur biblischen Vulgata, Hildesheim (reimpr.), r973, p. I62 s. 
3. Et maior (= magnus) seruiet minori (= paruo) (Gen. 25,23).

4. Non pugnauitis contra minorem (= paruum) et maiorem (= magnum) (I $R e .22,3 \mathrm{r}$ ).

5. Quod facis, fac citius (= cito) (Ioan. 13,27: griego).

\section{E) Superlativo por positivo.}

I. A minimo (= paruo) usque ad maximum (= magnum) (Ier. 44, I2). Véase esta misma fórmula traducida correctamente en Ier. 42, I: a paruo usque ad magnum. Y traducida, incorrectamente, por superlativos en 2 Cro. I5, I3; 34,30; Ier. 8, 10; Gen. I9, Ir; $A c^{t}$. 8, Io (en griego hay dos positivos); Iudit I3,I5 (en griego hay dos positivos).

2. A maximo (= magno) usque ad minimum (= paruum) (Est. $\mathrm{r}, 4)$.

3. A minimo (= paruo) usque ad magnum (I Sam. 30,2); cf. Ier. 42,8 .

\section{F) Superlativo por comparativo.}

I. Et accedens ad primum (= priorem) (Mat. 2I, 28,3I: dos hijos);

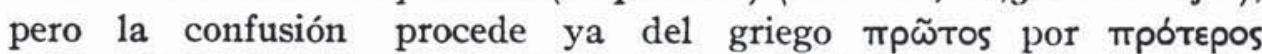

2. Primum (= prius) quidem sermonem feci (Act. I,I); pues Lucas sólo escribió dos obras; pero la confusión procede ya del griego, en donde $\pi \rho \tilde{T}$ tov está por трóтероv.

3. In nouissimis (= posterioribuः: v̇oTépoเs) temporibus discedent quidam (I Tim. 4, I).

4. Nouissime (= posterius: Ũ бтєроv) autem uenerunt duo testes (Mat. 26,6o); véase la misma expresión y el mismo intercambio en Mat. 21,37; Mar. 16,14; Luc. 20,32; Mat. 22,27.

Como puede verse, el latín bíblico ofrece un claro y bien palpable testimonio de todos los cambios, desplazamientos y desviaciones que sufrieron los grados de comparación del adjetivo en el latín tardío y que pueden verse ampliamente documentados en la Gramática latina de Leumann-Hofmann-Szantyr ${ }^{1}$. De todos los modelos analizados, el más alejado de las estructuras latinas clásicas, y que presenta por tanto una mayor novedad, es el modelo B 3 , I y 2, es decir, el grado positivo

1 Leumann-Hofmann-Szantyr, Lateinische Grammatik, II, Munich, 1972, pp. 162 ss., 168 ss. (cambio del superlativo y positivo; cambio del comparativo y positivo; cambio del comparativo y superlativo; positivo por comparativo y superlativo). 
con 1a construcción preposicional: magnus e fratribus suis; bellatorum fortium de filiis. E1 modelo consistente en el grado positivo y genitivo partitivo ( $\mathrm{C}_{3}, 2$ y 3 ) es más bien raro en latín: Ennio, Ann. 22, dia dearum; 64, sancta dearum; Virgilio, Aen. IV 576, sancte deorum. Es más frecuente con el grado positivo en plural: Salustio, Hist. 3,87: reliqua cadauerum; Livio XXVI 5,3: cum delectis peditum; XXVIII I4,I6: cum expeditis peditum equitumque; $\mathrm{XXX}$ 9, $\mathrm{r}$ : expeditis militum, etc. $\mathrm{y}$ otros ejemplos ${ }^{1}$. El modelo $\mathrm{C}_{3}, 2$ y 3 , por consiguiente, aunque raro en latín, no es totalmente nuevo. En cambio el modelo B 3, r y 2, de la Vetus Latina y de la Vulgata, atendiendo a todos los componentes del modelo (grado positivo y construcción preposicional) adquiere en latín el carácter de novedad absoluta, explicable solamente por el procedimiento del calco hebreo en la Vulgata y de propagación del calco

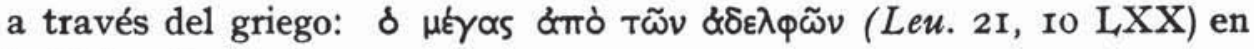
la Vetus Iratina ${ }^{2}$.

\section{Superlativo absol,uto.}

En hebreo (y arameo) el superlativo absoluto se expresa de varias maneras. Una de ellas, la más frecuente, consiste en poner detrás del adjetivo (o adverbio), siempre en grado positivo, la partícula mĕod, 'mucho', 'muy'.

\section{Traducción de la particula mëod.}

La partícula (adverbio) mĕod aparece en la Biblia hebrea unas 296 veces $^{3}$. La Biblia latina presenta aquí una gran variedad de traducciones, algunas de ellas sin importancia para nuestro tema, y de las cuales prescindiremos, pero otras importantes para comprender el superlativo y sus distintas sustituciones por adverbios de gradación o elevación.

1 Cf. Leumann-Hofmann-Szantyr, o. c., p. 55, $\S 52 ;$ A. Ernout-F. Thomas, Syntaxe latine 2 , Paris, 1964, p. $47 \mathrm{s.}$

a Cf. U. Rapallo, "Per una definizione diacronica e tipologica dei calchi ebraici nelle antiche versioni del Leviticon, en Atti del Istituto Lombardo (Rend. Lett.) 103, 1969, p. 383 .

3 Cf. S. Mandelkern, Veteris Testamenti Concordantiae hebraicae atque chaldaicae $_{7}$. Jerusalén, 1967, p. 652 (mě'od). A los textos de esta concordancia hay que añadir: Sal. 108 (I09), 30; Iud. 2, I5; Sal. II8 (I I9), I67 (bajo esta cita da, por error, el texto de Sal. I 8 (I I9), I40). 
Comenzando por las formas más representadas, podemos establecer el siguiente orden:

I. Nimis. Este adverbio aparece en la Biblia latina unas I44 veces ${ }^{1}$; de ellas, unas ro3 veces, aproximadamente, es la traducción del hebreo mĕ'od; otras 3 veces corresponde a textos traducidos del arameo (Dan. 3,22: fornax autem succensa erat nimis; Dan. 7,7: ...et fortis nimis; Dan. 7,I9: et terribilis nimis); otras 18 veces corresponde a textos traducidos del griego y es la traducción casi siempre de $\sigma \phi \delta \delta \rho \alpha^{2}$ y otras 20 veces es la traducción de distintas palabras hebreas o incluso el traductor lo añade $\sin$ correspondencia alguna con el hebreo ${ }^{3}$.

Comenzando el análisis por los textos en que nimis es la traducción del hebreo mĕ'od, hay unos 66 textos en que ese adverbio sirve al traductor latino para expresar el superlativo, acompañando a adjetivos (o adverbios) ${ }^{4}$. Las 37 veces restantes acompaña a verbos ${ }^{5}$.

Con respecto a las características de su empleo con adjetivos (o adverbios), podemos señalar lo siguiente: I. Va siempre detrás del adjetivo o adverbio, como en hebreo (pulchra nimis, magna nimis, stulte nimis, uelociter nimis, etc.), menos 3 veces, que va delante de la palabra, una en la frase: nimis profundae factae sunt cogitationes tuae (Sal. 9I(92),6: satis profundae en el Psalterium iuxta Hebraeos), siguiendo también en este caso el orden de palabras del texto hebreo y otros dos

1 Cf. F. P. Dutripon, Bibliorum sacrorum concordantiae ${ }_{\mathbf{8}}$, Hildesheim (reimpr. de 1880), 1976, p. 938 (nimis). Suprimir Sal. 95 (96), 4.

2 El adverbio $\sigma \varphi \delta \delta \rho \alpha$ aparece en los textos siguientes: Tob. 10,3; Iudit 2,10; 8,7; 10,7; 10,14; 12,20; Dan. 13,2; 13.31; I Mac. 1,4; 5,38; 16,7; 10,50; Eccle. 1,8; 17,23; corresponde a $\lambda \hat{i} \propto v$ en: $M a t .8,28 ;$ Mar. 9,2; no tiene correspondencia con el griego en: I Mac. 10,22; Eccli. 21,5.

s Ios textos son: Gen. 4I,2,18; 43,33; Ex. 9,31; II,9; Ios. II,4; Iud. 14,19; I Sam. 1,15; 2 Sam. 3,22; I Re. 10,18; I Cro. 23,17; 2 Cro. 2,9; Neh. 4,1,7; 5,2; 7,4; 11,14; Est. 2,7; Sal. 138 (139), 17 (dos veces).

- Los textos son: Gen. I2,I4; I3, I3; 15, I; 24, I6; Ex. 9,18; 12,38; Num. I1,33; Iud. 3,I7; II,33; 13,6; I Sam. 2,17; 4,10; 5,$9 ; 14,20 ; 18,15 ; 18,30 ; 25,2 ; 26,2$ I; 2 Sam. I,26; 3,8; 8,8; I2,5; I3,I5; 13,36; 14,25; 18,17; 19,32; 24,10; I Re. 4,29; I0,2; 10,10; I0,11; 14,26; 21,16; Is. 31,I; Ier. 2,36; I8,13; 24,2 (dos veces); 40,1 2; 48,16; Ez. 27,25; 40,2; 47,7; Ioel 2,1 I; Sof. 1,14; Sal. $47(48), 2 ; 78(79), 8 ; 91(92), 6$; 92(93),5; I18(119),96; 144(145),3; Iob. 1,3; Esdr. 10,1; Neh. 8,17; I Cro. 16,25; 2 Cro. 9,9; 32,29; Ez. 37,10; Ex. 1,7; Ez. 9,9; Ez. 16,13; I Sam. 25,36; I Re, 1,4; Dan. 8,8; Dan. II,25.

s Los textos son: Gen. 18,20; 47,27; Ex. 1,20; Num. 14,39; Zac. 9,5; Sal. 45 (46),2; 77(78),29; I06(I07),38; I08(I09),30; III(II2),2; I I 8(1 19),4,138; 138(139), 14; Iob. 8,7; Iud. 10,9; I Sam. 12,19; 14,31; 16,21; 17,11; 18,8; 28,5; 28,15; 2 Sam. 24,14; I Re. 1,15; 2,1 2; Neh. 2,2; 5,6; I Cro. 21,8; I Sam. I1,6; Sal. 96(97),9; I I5 (II6), I0; I4I(I42),7; Gen. 7,19; 17,2; Sal. 37(38),9; I Sam. I1,15; Ier. 50, I 2. 
en la fórmula acumulativa nimis ualde ( $E z .9,9 ; 37,10)$, que corresponde a la partícula hebrea repetida mĕ'od mĕod. 2) Hay un ejemplo de un adjetivo reforzado por la preposición prae-, acompañado además por el adverbio nimis: praeualidi nimis (Is. 3I, I); esta doble gradación es propia del traductor latino.

Respecto a los 20 textos traducidos del hebreo en los que nimis no corresponde a mĕod, advertimos lo siguiente: I. Acompaña a adjetivos I4 veces ${ }^{1}$ y 6 veces a verbos ${ }^{2}$. 2 . Va siempre detrás del adjetivo, como en los textos analizados antes.

En relación a los 18 textos traducidos del griego, señalamos lo siguiente: I. Nimis acompaña a adjetivos 12 veces ${ }^{3}$ y 6 veces a verbos ${ }^{4}$. 2. Va siempre detrás del adjetivo, menos una vez: Domus quae nimis locuples est (Eccli. 21,5). Este pasaje no tiene correspondencia en griego. 3. Nimis corresponde dos veces a $\lambda i \alpha v$ en el N. T.: saeui nimis (Mat. 8,28); candida nimis (Mar. 9,2), otras dos veces no tiene correspondencia con el griego (I Mac. 10,22; Eccli. 21,5), y las demás veces es la traducción de $\sigma \varphi \delta_{\delta} \rho{ }^{5}$.

En los 3 textos traducidos del arameo (Dan. 3,22; 7,$7 ; 7,19$ ), nimis corresponde al adverbio yatirah, 'mucho', 'muy', y va siempre detrás de la palabra que califica, como en arameo.

Para completar este análisis diremos por último que nimis va también siempre detrás del verbo, menos 4 veces: Sal. 96(97),9; Sal. I38(139), I7 (dos veces) y Eccli. I7,23, que va delante; pero tanto en el primer caso como en el último, el orden de palabras corresponde al del texto hebreo y griego respectivamente.

2. Valde. Este adverbio aparece en la Vulgata unas 159 veces ${ }^{6}$; de ellas, 70 veces es la traducción de mĕod; una vez se encuentra en Dan. 7,19: dissimilis ualde ab omnibus, sin correspondencia con el arameo; otras 64 veces corresponde a textos traducidos del griego, y en

\footnotetext{
1 Los textos son: Gen. 4I,2; 4I,18; Ex. II,9; Ios. II,4; I Sam. I,15; 2 Sam. 3,22; I Re. I0,18; 2 Cro. 2,9; Neh. 5,2; 4,I; 7,4; I 1,14; Est. 2,7; Iud. 14,19.

2 Los textos son: Gen. 43,33; Ex. 9,35; I Cro. 23,17; Neh. 4,7; Sal. 138(139), I 7 (dos veces).

3 Los textos son: Iudit 2,10; 8,7; 12,20; Dan. 13,2; 13,31; I Mac. 1,4; 5,38; 16,7; Eccli. 21,5; Eccli. 1,8; Mat. 8,28; Mar. 9,2.

- Los textos son: Tob. 10,3; Iudit 10,14; I Mac. 10,22; 10,50; Eccli. 17,23; Iudit 10,7.

s Véanse los textos en p. 353, n. 2.

- Cf. F. P. Dutripon, o. c., p. I 410 (ualde). A esta lista hay que añadir el Sal. 95(96),4, según la edición critica del Salterio.
} 
éstos, 5I veces traduce a $\sigma \phi \delta ́ \delta \rho \alpha^{1}, 8$ veces a $\lambda i \alpha v^{2}$, una vez a $\pi{ }^{1} v v$; 2 Mac. 15,17: sermonibus bonis ualde; una vez a eṽ $\mu \alpha \dot{\lambda} \lambda \alpha$ : 2 Mac. 10,18: turres valde munitas; 2 veces no tiene correspondencia con el griego: Est. 15,17: ualde enim mirabilis ${ }^{3}$, y una vez a ßatús: L.uc. 24,I:

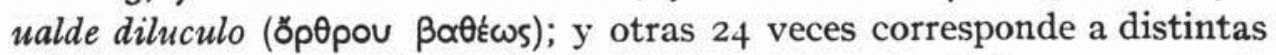
palabras hebreas o el traductor lo añade por su cuenta ${ }^{4}$.

Comenzando el estudio por los 70 textos en que ualde corresponde al adverbio hebreo mĕ'od, señalamos que $4 \mathrm{I}$ veces acompaña a adjetivos (o adverbios) y las 29 veces restantes a verbos ${ }^{5}$.

Las características de su empleo con adjetivos y adverbios pueden resumirse así: r. Valde va siempre detrás de la palabra como en hebreo, (diues ualde, magnus ualde, procul ualde, iuxta ualde), menos en 1 os 5 textos que citamos a continuación, en que va delante, aunque en hebreo la partícula correspondiente a ualde va también detrás:

I. Erant ualde bona (Gen. I,3I).

2. Pestis ualde grauis (Ex. 9,3).

3. Terram ualde opulentam (Iud. I8,9).

4. Erubescant ualde velociter (Sal. 6,II: confundantur subito en el Psalterium iuxta Hebraeos).

5. Terra ualde bona (Num. I4,7: el hebreo repite mĕod mĕ'od; cf. Agustín, Locut. Hept. Num. 38: valde ualde bona).

1 Las textos son: I) con adjetivos: $\operatorname{Dan}$. I3,4; I Mac. I,6; $3,27,4 \mathrm{I} ; 4,58 ; 5,45$, 46; 6,2,41; 8,4,6; 9,22,24; 10,2; 13,22; 2 Mac. 3,11; Eccli. 39,21; 43,1 2; 47,30; Mat. 2,10; 26,22; Mar. 16,4; Luc. 18,23; 2) con verbos: Iudit 4,$1 ; 8,8 ; 14,17 ; 15,7 ; 1$ Mac. 2,14,39; 3,31; 4,2I; $5,1,63 ; 6,8 ; 7,48 ; 8,13 ; 9,6,68 ; 10,46,68 ; 11,53 ; 12,52 ; 13,49$; 14,16; Eccli. 7,19; 25,3; Mat. 17,6; 18,31; 19,25; 27,54; Act. 6,7.

2 Mat. 2,16; 4,8; Mar. 1,35; 16,2; Luc. 23,8; 2 Tim. 4, 15; 2 Io. 4; 3 Io. 3.

3 Eccli. 46,6: uirtutis ualde fortis, también aquí está añadido el ualde.

- Los textos son: I) con adjetivos: Gen. 27,15; 36,7; Ex. 32,19; Ios. 7,21; I Re. 6,33,35; I Cro. 4,40; 15,22; Est. I, I I; 2,15; 2) con verbos: Gen. 32, I ; 34,19; 39,19; Iud. 4,19;6,2;8,5;9,30; 21,$16 ; 2$ Sam. 13,2; Est. 3,5; 5,9; 2 Cro. 16,10; Neh. 5,$18 ; 2,2$.

- Los textos son: I) con adjetivos: Iud. I5,18; Gen. 1,31; 13,2; 26,16; Ex. 9,3; I1,3; Num. 16,15; Deut. 20,15; 28,54; 30,14; Ios. 1,7; 9,9; 9,22; Iud. 18,9; I Sam. 2,22; 5,$12 ; 19,4 ; 2$ Sam. 10,5; I1,2; 12,2; 12,30; 13,3; 19,32; I Re. 1,6; Is. 16,6; 52,13; Ier. 24,3 (dos veces); 48,29; Ez. 37,2; Ioel 2, I I; Ab. 2; Zac. 14,4; Sal. 6,1 I; Sal. 95(96),4; Neh. 13,8; 2 Cro. 7,8; 32,27; Num. 14,7; Ez. 37,10; Ez. 9,9; 2) con verbos: Gen. 20,8; 24,35; 32,7; 34,7; Ex. 14,10; Num. 11,10; Is. 47,6; Nah. 2,2; Zac. 9,2; Sal. 30(31),12; 77(78),59; Iob 35,15; Ios. 9,24; 10,2; I Sam. 17,24; 19, I; 21,12; 2 Sam. 24,10; I Re. 5,7; 11,19; 18,3; Rut. 1,20; 2 Cro. 33,12; Iud. 6,6; I Sam. 28,21; I Sam. 30, 6; 2 Sam. 13,21; Sal. 6,4; Gen. 17,20. 
2. Hay un caso de comparativo con ualde, que es una redundancia del traductor latino: Potentior nostri (o nobis) factus est ualde (Gen. 26, I6). 3. Hay 2 casos de superlativo orgánico, acompañados del adverbio de intensidad, que son una redundancia del traductor latino: I Sam. 5,12: grauissima ualde manus Domini; 2 Sam. 12,2: boues plurimos ualde. 4. La redundancia de nimis ualde es, como dijimos, una traducción literal del hebreo mĕ'od mĕ'od: Ez. 9,9: iniquitas... magna est nimis ualde; Ez. 37,10: exercitus grandis nimis ualde.

Respecto a la colocación de ualde con verbos en este bloque de textos, debemos señalar que ualde va siempre detrás del verbo (timuit ualde, etc.), como en hebreo, menos una vez, Rut. $\mathrm{r}, 20$ : amaritudine ualde me repleuit Omnipotens, en que va delante, en contra del orden hebreo.

Con respecto a los 24 pasajes, traducidos del hebreo, en los que ualde no corresponde a mĕod, tenemos que señalar lo siguiente: I. Ese adverbio acompaña a adjetivos (o adverbios) ro veces ${ }^{2}$ y I4 veces a verbos $^{1}$. 2. Acompañando a adjetivos (o adverbios) va 6 veces delante de la palabra y sólo 4 veces detrás (Gen. 36,7; Ex. 32,I9; Est. I,II; 2,I5). Esste orden de palabras, en contraste evidente con el otro bloque de textos analizados antes, no deja de ser sorprendente. Ahora bien, comparando todos estos pasajes con el correspondiente texto hebreo, encontramos que el adverbio ualde es una adición propia del traductor latino, ya que en el texto hebreo no hay ninguna palabra que la apoye. Podemos, pues, suponer que el traductor, liberado aquí del orden fijo de palabras en el texto hebreo (adjetivo y partícula de gradación pospuesta: magnus ualde), pone en latín el orden que más le conviene, adverbio de gradación y adjetivo (ualde magnus). 3. Respecto al orden de ualde con verbos sucede algo parecido: 5 veces va delante (ualde timeo, etc.) y ninguna de ellas tiene correspondencia con el hebreo, es decir, que ualde es una adición del traductor latino ${ }^{3} ; 9$ veces va detrás ", conservando así el orden normal que hemos descubierto en el bloque de textos en que aparece mĕod, pero también aqú las 9 veces

Véase p. 355, n. 4 .

Véase p. 355, n. 4.

s Ios textos son: Gen. 32,11; Iud. 8,5; 21,15; 2 Cro. 16,10; Neh. 5,18.

- Los textos son: Gen. 34,19; 39,19; Iud. 4,19; 6,2; 9,30; 2 Sam. 13,2; Est. 3,5; 5,9; Neh. 2,2; sólo este último texto tiene algún apoyo para el ualde (harbeh); el texto completo dice: et timui ualde ac nimis (mĕ'od); el traductor podía haber puesto, con más exactitud: nimis ualde o uehementer nimis, fórmulas que ha usado otras veces: Gen. 17,2 y Ez. 16,13 (uehementer nimis); Ez. 37, 10 y Ez. 9,9 (nimis ualde). 
ualde es una adición propia del traductor latino sin correspondencia con el hebreo.

En los libros traducidos del griego, ualde aparece, como dijimos, 64 veces; $3 \mathrm{I}$ de ellas con adjetivos y 33 con verbos. Respecto a su empleo con adjetivos, podemos anotar los siguientes datos: I. Va delante del adjetivo (ualde fortis, etc.) 7 veces; 2 de ellas no tienen correspondencia con el griego, es decir, ualde es una adición propia del traductor latino: Est. 15,I7: ualde enim mirabilis; Eccli. 46,6: ualde fortis; 3 veces sigue el mismo orden que el texto griego; Eccli. 43, I2: ualde speciosus; 2 Mac. 3,II: ualde eminentis; Mar. I6,2: ualde mane; las 2 veces restantes el traductor latino sigue el orden contrario al del texto griego: $2 \mathrm{Mac}$. I0,18: ualde munitas; Luc. 24, I: ualde diluculo. 2. Va detrás del adjetivo 24 veces (magna ualde, etc.) ${ }^{1}$, coincidiendo este orden con el del texto griego todas las veces menos una (2 Mac. 15,17: sermonibus bonis ualde), en que móvv va delante y en latín ualde va detrás del adjetivo. 3. Hay en latín un superlativo orgánico reforzado por el adverbio valde: plurima... peccata ipsorum ualde (Eccli. 47,30), redundancia propia del traductor latino, sin apoyo en el texto griego.

Respecto a la colocación de ualde con verbos en los textos traducidos del griego, podemos anotar lo siguiente: I. Valde va delante del verbo una sola vez: ualde enim restitit (2 Tim. 4,I5), coincidiendo este orden con el del texto griego. 2. Las 32 veces restantes va detrás del verbo, coincidiendo siempre con el texto griego ${ }^{2}$. 3. En Judil 4, I, el traductor latino pone timuerunt ualde, suprimiendo uno de los dos $\sigma \phi \delta \delta \rho \alpha$.

3. Vehementer. Este adverbio aparece en la Vulgata $5+$ veces $^{3}$; de ellas, 28 veces es la traducción de mè'od; una vez se encuentra en IJan. 6,23: uehementer gauisus est y corresponde al adverbio arameo saggi $i$, 'mucho', 'muy'; otras $\mathbf{I}_{5}$ veces corresponde a textos traducidos del griego y en éstos, 7 veces traduce a $\sigma \phi \delta \delta \rho \alpha^{4}$ y las 8 veces restantes a otros términos griegos o no tiene correspondencia alguna con el griego ${ }^{5}$;

1 Los textos son: Dan. 13,4; I Mac. I,6; 3,27,4 I; 4,58; $5,45,46 ; 6,2,4$ I; $8,4,6$; 9,22,24; 10,2; 13,22; Eccli. 39,21; 47,30; Mat. 2,10; 4,8; 26,22; Mar. 1,35; 16,4; Luc. 18,23; 2 Mac. 15,17.

2 Los textos son: Iudit 4,I; 8,8; I4, I7; 15,7; I Mac. 2,14,39; 3,3I; 4,21; 5, I,63; 6,8; 7.48; 8,13;9,6,68; 10,46,68; 11,53; 12,52; 13,49; 14,16; Eccli. 7,19; 25,3; Mat. 2,16; 17,6; 18,31; 19,25; 27.54; Luc. 23,8; Act. 6,7; 2 Io. 4; 3 Io. 3 .

3 Cf. F. P. Dutripon, o. c., p. I4I4 (uehementer).

- Los textos son: Eccli. 43,41; 51,32; Dan. 13,27; 14,29; I Mac. 16,32; Mat. 17,22; Apoc. 16,21.

- Los textos son: Iudit 6, I (sin correspondencia); Dan. 14,27 ( $\lambda(\alpha v) ; 2$ Mac. 
y otras Io veces corresponde a distintas palabras hebreas o el traductor latino lo añade por su cuenta sin apoyo en el texto ${ }^{1}$.

Vehementius aparece en la Vulgata 2 veces; una, como traducción de më'od: Ex. 19,16: clangor buccinae uehementius strepebat, y otra, sin correspondencia alguna con el hebreo, siendo, por consiguiente, una adición del traductor latino: Num. 22,27: uehementius caedebat.

Vehementissime se encuentra en la Vulgata 3 veces; dos de ellas como traducción de mëod: Gen. I9,9: uimque faciebant Lot uehementissime; Gen. I7,6: faciamque te crescere uehementissime, siendo en este último caso la traducción de mĕ'od mĕ'od, la partícula hebrea repetida. La tercera vez aparece en 2 Cro. I3,18: uehementissime confortati, sin correspondencia alguna con el hebreo, siendo, por tanto, una adición del traductor latino.

Pasando al análisis de los textos en que uehementer corresponde al adverbio mĕ'od ( 28 veces en total), observamos que acompaña a adjetivos (o adverbios) 8 veces y las 20 veces restantes a verbos. El uso de uehementer con adjetivos presenta las siguientes características: I. Va 6 veces detrás del adjetivo (sicca uehementer, etc.), como en hebreo ${ }^{2}$, y sólo 2 veces delante, aunque en hebreo también estas 2 veces va detrás: Deut. 9,20: uehementer iratus; 2 Cro. 25, 10: uehementer irati. 2. Hay un caso de redundancia de adverbios, uehementer nimis, que es una traducción literal del hebreo mĕ'od mĕod: Ez. 16,13: decora facta es uehementer nimis. 3. Hay, además, un superlativo orgánico acompañado del adverbio de intensidad: Ier. 14,I7: plaga pessima uehementer; es una redundancia propia del traductor latino sin apoyo en el texto que sólo tiene un positivo, mala.

Cuando uehementer acompaña a verbos, va detrás del verbo 18 veces (considerate uehementer, etc.) ${ }^{3}$, como en hebreo, y sólo 2 veces de-

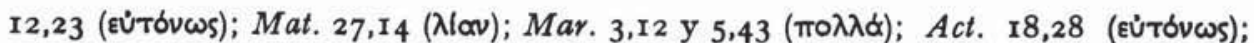
Fil. 4, 10 ( $\mu \varepsilon \gamma \dot{\alpha} \lambda \omega s)$.

1 Vehementer no tiene apoyo alguno en el texto en los casos siguientes: Gen. 43.1; Iud. 4,3; 10,8; I Sam. 1,6; Iob 32,5; Prou. 30,33; tiene algún apoyo en los siguientes casos: I Cro. 4,38: multiplicati sunt uehementer (larob); Esdr. I0,13: uehementer (hirbinü) quippe peccauimus; Iob 3,22: gaudent uehementer (literalm. gaudent gaudio); Lam. 1,9: deposita est uehementer (literalm. deposita est mirabiliter).

2 Los textos son: Ez. 37,2; Sal. 118(119),140; Gen. 26,13; Ier. 9,18; 14,17; Ez. 16,13.

3 Los textos son: Gen. 4,5; Iud. 2,15; I Sam. 31,3; 2 Re. 17,18; Ier. 2,10; 2,12; 20,1 I; 49,30; Ez. 20,13; Sal. 20(2I),2; 104(I05),24; II8 (II9),167; 2 Cro. 33,14; Sal. 103(104),1; 2 Re. 10,4; Gen. 17,2; Lam. 5,22; Is. 64,1 2. 
lante, una de ellas coincidiendo con el hebreo: Sal. 46(47), ro: dii... uehementer eleuati sunt (scuta... uehementer cleuata sunt en el F'salt. iuxta $H e b r$.), y la otra, en contra del texto hebreo, que lleva el adverbio detrás: Gen. 7,18: uehementer enim inundauerunt. Hay también aquí un caso de redundancia de adverbios, uehementer nimis, que se debe a la traducción literal del hebreo mĕ'od mĕod: Gen. I7,2: multiplicabo te uehementer nimis.

Con respecto a los ro pasajes, traducidos del hebreo, en que uchementer no corresponde a mě od, cabe señalar lo siguiente: I. Sólo acompaña a adjetivos una vez: Iud. Iо,8: uehementer oppressi, y es una adición del traductor latino. 2. De las 9 veces restantes en que acompaña a verbos, va delante del verbo 5 veces ${ }^{1}$ y es siempre una adición del traductor latino sin apoyo real en el texto; 4 veces va detrás del verbo y todas, menos una (Iob. 32,5: iratus est uehementer), tienen alguna justificación, por ir en hebreo detrás del verbo alguna palabra que significa 'mucho' 2 .

En relación a los 15 textos traducidos del griego, anotamos los siguientes detalles: r. Vehementer acompaña a adjetivos 3 veces, y las 3 va detrás del adjetivo, pero una vez no tiene correspondencia con el griego, siendo, por tanto, una adición del traductor latino: Iudit 6,I: indignatus Holofernes uehementer; otra vez sigue un orden contrario al griego (en griego, delante del adjetivo; en latín, detrás): Eccli. 43,3r: et magnus uehementer; y una vez coincide con el griego: Apoc. I6,21: magna facta est uehementer. Fin estos dos últimos textos la palabra griega que traduce uehementer es $\sigma \phi \delta \delta \delta \rho \alpha$. 2. Vehementer acompaña a verbos I2 veces. Va delante del verbo 4 veces, 2 de ellas coincidiendo con el

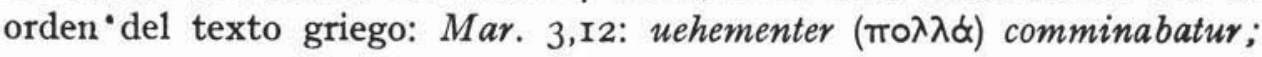

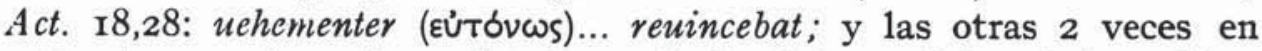
orden inverso (en griego, detrás; en latín, delante del verbo): I Mac. 16,22: uehementer ( $\sigma \phi \delta \delta \rho \alpha)$ expauit; 2 Mac. 12,23: uehementer instabat

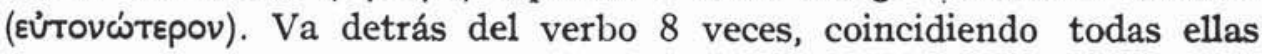
con el orden del texto griego ${ }^{3}$.

4. Satis. Este adverbio aparece en la Vulgata 20 veces ${ }^{4}$; de ellas, 6 corresponden a la traducción de mĕ’od; en este caso, 4 veces acompaña

1 Los textos son: Gen. 43,I; Iud. 4,3; I Sam. 1,6; Esdr. 10,13; Prou. 30,33.

Véase, no obstante, p. 358 , n. I.

2 Los textos son: I Cro. 4,38; Iob 3,22; Lam. I,9.

- Los textos son: Eccli. 51,32; Dan. 13,27; 14,27; 14,29; Mat. 17,22; 27,14;

Mar. 5.43; Fil. 4,10.

- Cf. F. P. Dutripon, o. c., p. 1235 (satis).

8 
a adjetivos y va siempre detrás del adjetivo, como en hebreo: I Sam. 25, I5: homines isti boni satis; 2 Sam. 2,17: bellum durum satis; Ez. 47,9: pisces multi satis; Zac. 14,I4: uestes multae satis. Dos veces acompaña a verbos y va detrás del verbo, como en hebreo: Zac. 9,9: exsulta satis; Is. 64,9: ne irascaris, Domine, satis. La colocación del adverbio satis en este bloque de textos depende evidentemente del influjo hebreo.

Otros 2 textos con satis se encuentran en las partes arameas del libro de Daniel. Aqui el adverbio satis va delante del verbo: Dan. 5,9: satis conturbatus est; Dan. 6,14: satis contristatus est. Fin estos dos casos, el orden puede depender también del texto original, pues en arameo el adverbio saggī, 'mucho', va también delante del verbo.

En los libros de la Biblia traducidos del hebreo, el adverbio satis aparece aún otras 4 veces: va siempre delante del verbo o adjetivo: Leu. 13,28: candor... non satis clarus; 2 Sam. 18,3: non satis curabunt; Eccle. 5,19: non satis recordabitur; Ez. 34,I8: nonne satis uobis erat...?'; pero en ningún caso tiene apoyo en el texto original, es decir, se trata siempre de adiciones propias del traductor latino.

En los libros traducidos del griego satis aparece 8 veces: va siempre delante del verbo o adjetivo (o adverbio). Acompañando a adjetivos tenemos los siguientes pasajes: Tob. 10,6: satis fidelis (el adverbio sin correspondencia con el griego); 2 Mac. 9,6 y 13,8: satis ( $\pi \dot{\alpha} v \mathrm{v}$ ) iuste (el mismo orden en griego); 2 Mac. 10,19: satis multis (el adverbio sin correspondencia con el griego). Acompañando a verbos hay también 4 textos: I Mac. 7,2I: satis agebat y 2 Mac. 7,42: satis dictum est (en ambos casos el adverbio es una adición del traductor latino); Luc. 22,38: satis est y Act. 20,II: satis allocutus... (en ambos casos ikavóv y el mismo orden en griego).

5. Superlativos orgánicos. Además de los cuatro adverbios citados, la Biblia latina utiliza superlativos orgánicos para traducir el mĕ’od hebreo. Ya citamos antes los casos de uehementissime. Vamos a mencionar ahora otros superlativos que el traductor latino podía haber reproducido con un positivo y alguno de los cuatro adverbios citados. Encontramos los siguientes superlativos: uentum uehementissimum (Ex. I0,I9); uir mitissimus super omnes (Num. I2,3); terra latissima (Ios. 13,I); diligentissime praecaute (Ios. 23, II); languor fortissimus (I Re. I7,I7); auri plurimum (2 Cro. 9, I); aeris plurimum (I Cro. 20,2); manubias plurimus (I Cro. 20,2); summa diligentia (2 Cro. II,I2).

6. Otros modos de traducción. Para completar en lo posible el análisis de la traducción de mĕ’od, y demostrar al mismo tiempo la libertad 
con que actuaban los traductores latinos de la Biblia, vamos a considerar algunos otros ejemplos de traducción del mëod hebreo.

a) Adjetivos en grado positivo: Immensus: immensa pondera (Deut. I7,I7); immensae multitudinis (Ios. II,4); uehemens: planctu uehementi (Gen. 50,10); disceptatio uehemens (Iud. I2,2); duritiam uehementem (Is. 47,9); dolorem uehementem (Iob 2,I3); stupore wehementi; nimius: ambitione nimia (2 Cro. I6,I4); nimio terrore (I Sam. 31,4); multitudinem nimiam (I Re. 7,47); multitudinis nimiae (Ez. 47,10); multitudine nimia (2 Cro. I6,8); nimio furore (Est. I, I2); multus: populi multi (2 Cro. 30,13); multo cum comitatu (I Re. 10,2); spolia multa (2 Cro. I4,I2); multae miserationes (I Cro. 2r, $\mathrm{r} 3$ ); infinitus: infinita substantia (Num. $32, \mathrm{I})$; infinitam multitudinem (2 Cro. 24,24); innumerabilis: locustae innumerabiles (Ex. I0,I4); multitudo innumerabilis (2 Cro. 4,I8); multiplex: ueste multiplici (Ios. 22,8); terribilis: mons terribilis (Ex. I9,I8); magnus: plaga magna (Ios. 10,20); festinus: descende festinus (I Sam. 20,I9); ualidus: tempestas ualida (Sal. 49(50),3); grandis: grandem contumeliam (I Cro. 19,5); urbes grandes (Num. 13,29); innumerus: oppidis innumeris (Deut. 3,5). En todos estos casos, el traductor suple la idea de gradación que existe en el adverbio mĕod cón estos adjetivos, la mayoría de los cuales encierran una idea superlativa.

b) Adverbios: Oppido: oppido unlneratus sum (2 Cro. 35,23); compulit eos oppido (Gen. 13,3); diligenter: uidete diligenter (Deut. 2,4); obserua diligenter (Deut. 24,8); ultra modum: ditatus est ultra modum (literalmente: ualde ualde) Gen. 30, 43; sollicite: custodi sollicite (Deut.4,15); attente: custodiatis attente (Ios. 22,5); magis: magis me premit (Rut. I, I3); usquequaque: humiliatus sum usquequaque (Sal. II 8 (II 1 ), 8, 43, 51, I07; prolixius: prolixius tendebatur (Ex. 19,I9); amplius: ut multipliceris amplius (Deut. 6,3), multo amplius (Is. 56,13); longius: nec longius recedatis (Ios. 8,4; cf. Ios. 9,13: longioris uiae). También en estos casos, el traductor intenta suplir la idea de gradación con estos adverbios.

c) Omisión total de mëod. En muchos casos el traductor latino omite simplemente la traducción de mĕod o da una versión ad sensum ${ }^{1}$.

1 Casos de omisión pueden considerarse los siguientes: Gen. 34, 12: augete dotem; Gen. 41,19: in tantum deformes... ut; Gen. 4I,3I: inopiae magnitudo (trad. libre); Gen. 41,49: ut arenae maris coaequaretur (trad. libre); Gen. 47,13: et oppresserat fames terram (trad. libre); Gen. 50,9: turba non modica (literalm. magna nimis); Ex. 9,24: tantaeque fuit magnitudinis; Num. 22,3: pertimuissent; Num. 22,17: paratus sum honorare te (om. ualde); Ios. 3,16: procul ab urbe (literalm. procul ualde...); Ios. 23,6: confortamini (om. ualde); Iud. I9, II: dies mutabatur in noctem (literalm. descendebat ualde); I Sam. 28,20: extimuerat (om. ualde); I Re. 21,26: abominabilis factus est (om. ualde); Est. 4,4: consternata est (om. ualde); Neh. 6,16: 
2. Repetición del mismo nombre en plural.

E1 segundo tipo de superlativo absoluto hebreo (y arameo) ${ }^{1}$ consiste en la repetición del mismo nombre en plural. Cuando se trata de adjetivos, el adjetivo irá en grado positivo, seguido del genitivo plural del mismo adjetivo. Si se trata de sustantivos, el sustantivo irá seguido de su propio genitivo plural ${ }^{2}$. Cuando los dos nombres están en singular aunque la regla que acabamos de exponer no se aplica con todo rigor, también ese conjunto tiene sentido superlativo en hebreo.

He aquí algunos ejemplos, con su posible traducción al español:

I. Sanchum sanctorum ('el lugar santísimo') (Ex. 26,34; Leu. 6, I7; 7,6 ; 10,12; 10,17; 27,28).

2. Sanctum sanctorum (de) ('la cosa o la parte sacratísima [de]') (Leu. 2,3; 2,10; 24,9).

3. Sancta sanctorum ('las partes o las cosas sacratísimas') (Leu. 6,I7 VL; I0,I2 VL; IO,I7 VL; I4,I3 Vg).

4. Canticum canticorum ('el Cantar [por excelencia]') (Cant. I, I).

5. Vanitas uanitatum ('vanidad suma, suprema') (Eccle. $\mathrm{I}, 2$ [dos veces $]$; 12,8$)$.

6. Seruus seruorum ('el último siervo') (Gen. 9,25) ${ }^{3}$.

7. Princeps principum ('el príncipe supremo') (Num. 3,32; Dan. $8,25)$.

8. Principes principum ('los príncipes supremos') (Ez. 23,33).

9. Deus deorum ('el Dios supremo, único') (Deut. Io, I7; Sal. 49(50), I; 84(85),8; I35(r36),2; Dan. 2,47; 3,90; II,36).

ro. Dominus dominorum ('el Señor supremo, único') (Sal. r35(r36), 3 у 26$)$.

II. Dominus dominantium ('el Dominador supremo, único') (Deut. 10,17; I Tim. 6,15; Apoc. 19,16).

12. Caeli caelorum ('los cielos más altos') (I Re. 8,27; Eccle. I6, I7 (18); 2 Cro. 2,6; 6,18; Sal. I48,4).

conciderent (om. ualde) intra semetipsos; I Cro. 10,4: timore perterritus (om. ualde); I Cro. 21,3: me angustiae premunt (om. ualde); I Cro. 21,13: multae (om. ualde) sunt miserationes eius. Hemos puesto entre paréntesis ualde, como podriamos haber puesto nimis o uehementer.

1 Cf. P. Joüon, o. c., p. 438, § I4 I I; L. Palacios, Grammatica aramaico-biblica, Roma, 1953a, p. 72. Se trata esencialmente de un superlativo absoluto, aunque en algún caso, si le sigue un complemento nominal con la preposición min, pueda considerarse como un superlativo relativo; cf. U. Rapallo, l. c., p. 384, n. 26.

2 Cf. P. Joüon, o. c., p. 438, § I4I I; L. Palacios, ibidem.

s Título aplicado posteriormente al Papa. 
I3. Caelum caelorum ('el cielo más alto') (Neh. 9,6).

I4. Caelum caeli ('el cielo más alto') (Sal. 67(68),34; II3(II5), I6; Deut. I0, I4).

15. Rex regum ('el rey supremo') (Dan. 2,37 [arameo]; Ez. 26,7; I Tim. 6, I5; Apoc. 19,16).

16. Saecula saeculorum ('los siglos [venideros] más remotos') (Tob. 8,9; 13,23; Is. 34,10; Rom. 16,27; Gal. 1,5; Fil. 4,20; r Tim. 4,17; 2 Tim. 4,I8; Hebr. I3,2I; I Pe. 4,II; 5,II;Apoc. I,6,I8; 4,9, I0; 5,I3,I4; 7,I2; Io,6; II,I5; I4,II; I5,7; I9,3; 20,10; 22,5).

17. Omnia saecula saeculorum ('todos los siglos [venideros] más remotos') (Tob. I3, I2; Iudas 25$)^{1}$.

18. Saeculum saeculi ('el siglo [venidero] más remoto') (Is. 45,17; Sal. 9,6; 9(ro),16; I8(I9), 10; 20(2I),5,7; 21(22),27; 36(37),27,29; 44(45), $7, \mathrm{I} 8 ; 47(48), \mathrm{I} 5 ; 5 \mathrm{I}(52), 10 ; 60(6 \mathrm{I}), 9 ; 82(83), \mathrm{I} 8 ; 88(89), 30 ; 83(84), 5 ; 9 \mathrm{I}(92)$, 8; I03(IO4),5; IIO(III),3,8, I0; $\operatorname{III}(\operatorname{II} 2), 3,9 ; \operatorname{II} 8(\operatorname{IIg}), 44 ; \operatorname{I3I}(\operatorname{I32}), \mathrm{I} 4$; I44(I45),I,2,2I; I48,6; 2 Cor. 9,9; Hebr. I,8.

19. Generationes saeculi saeculorum ('las generaciones de los siglos [venideros] más remotos') (Ef. 3,2I).

20. Generationes generationum ('las generaciones [venideras] más alejadas') (Is. 5I,8).

21. Dies generationis et generationis ('la fecha de la generación [venidera] más alejada') (Sal. 6o(6r),7; Is. 58, r2; Ioel 2,2 ${ }^{2}$.

E1 latín arcaico presenta estructuras parecidas a este tipo de superlativo hebreo. Ya el elogio de Lucio Esscipión tiene la fórmula: duonoro $(\mathrm{m})$ optumo $(m)$ y Nevio y Plauto usan las expresiones: magnorum maximus y pessimorum pessimus, expresiones que reaparecen luego en el latín postclásico con Apuleyo, Met. XI 30,3: summorum maximus ${ }^{3}$. Pero hay que advertir que estas fórmulas no son totalmente idénticas a las de

1 Cf. la conocida fórmula litúrgica con que terminan las oraciones: Per omnia saecula saeculorum. Otro conocido ejemplo es la fórmula de la letanía de la Virgen: Virgo Virginum.

2 Esta fórmula reviste una gran variedad de formas, aunque sólo la citada en el núm. 20 reúne las condiciones exigidas para constituir el superlativo de que venimos tratando. Estas formas son: a generatione in generationem (Is. 34, 10; Eccli. 39, 13; Sal. 9(10),6; 76(77),9; 84(85),6; in generatione et generationem (Sal. $32(33), 11 ; 44(45), 18 ; 71(72), 5) ;$ generatio et generatio (Sal. 144(145),4); in omni generatione et generatione (Sal. $\left.\mathrm{I}_{44}(\mathrm{1} 45), \mathrm{r} 3\right)$; in generationem et generationem (Lam. 5,19; Dan. 3,100; 4,30; Ioel 3,20; Prou. 27,24; Eccli. 44, 14; Is. 34,17; 60, I5; 61,4; Sal. 78(79),13; 88(89),2,5; 99(100),5; I01(102),13,25; 105(106),31; 118(119), 90); ad generationem et generationem (Is. 13,20; Ier. 50,39); per generationem et generationem (I Mac. 2,61).

3 Cf. otros ejemplos en Leumann-Hofmann-Szantyr, o. c., p. $54, \S 52$ b. 
la Biblia latina, puesto que en el latín bíblico se trata de positivos (sanctum, sancta) o sustantivos, en cambio, en el latín profano, de superlativos (maximus, pessimus).

Con este tipo de fórmulas bíblicas se relaciona el llamado genitivo de gradación, elevación o ponderativo ${ }^{1}$, que consiste, como en hebreo, en la unión de un sustantivo con el genitivo plural - raras veces singular- del mismo sustantivo. El tipo fraseológico, regum rex, documentado ya desde Plauto (Capt. 825) y que continúa a través de toda la tradición latina (Cic., Epist. IX 14,2; Liv. XLV 27,9, etc.), sin olvidar que ya el Carmen Saliare conoce la expresión diuom deo, es un tipo de genitivo común al antiguo indio, antiguo persa y céltico ${ }^{2}$.

Los autores no están de acuerdo sobre el origen de este genitivo. Unos le atribuyen origen semítico, otros, origen popular, dado que la lengua popular siempre tiende a la amplificación afectiva ${ }^{3}$.

Sin entrar a fondo en este tema, por lo que respecta al latín bíblico, podemos afirmar sin género de duda que las fórmulas que nos ocupan son siempre una traducción literal del hebreo (o arameo) y que por tanto se explican por influjo semítico, al menos en cuanto a su frecuencia y uniformidad. Hasta el orden de palabras en las fórmulas - posposición constante del genitivo, rex regum - obedece a influjo semítico; en cambio, en el latín profano el genitivo puede ir delante o detrás: regum rex (Plauto, Capt. 825); uictor uictorum (Plauto, Trin. 309).

En las fórmulas del latín profano puede tratarse de un genitivo partitivo, 'rey de los reyes', no posesivo, 'gran rey'; pero las fórmulas del latín bíblico tienen sentido superlativo en dependencia del superlativo absoluto hebreo (y arameo) 4. Las traducciones literales de la Biblia han influido en la conservación y propagación de algunas de estas fórmulas en las lenguas románicas. En español es normal decir Cantar de los cantares; por los siglos de los siglos; el santo de los santos; vanidad de vanidades; Señor de los señores, etc. El sentido superlativo de estas frases queda muchas veces oscurecido o no se percibe en todo su valor.

Llegados al final de nuestro análisis de los textos, vamos a recoger en sintesis algunas conclusiones:

\footnotetext{
1 Cf. Leumann-Hofmann-Szantyr, o. c., p. $55, \S 52$ g; M. Bassols, Sintaxis latina, I, Madrid, I963, p. 86.

2 Cf. Leumann-Hofmann-Szantyr, o. c., p. $55, \S 52 \mathrm{~g}$

3 Cf. Leumann-Hofmann-Szantyr, ibidem; M. Bassols, o. c., p. 86

- No compartimos la opinión de Trost, ZfSem. Io, I935, p. 326 ss. (citado por Leumann-Hofmann-Szantyr, ibidem), de que también en semítico se trata de un genitivo partitivo.
} 
I. E1 latín bíblico ofrece un testimonio evidente de los cambios sufridos por los grados de comparación del adjetivo en el latín vulgar y tardío. Las interferencias entre los distintos grados del adjetivo son constantes y apuntan hacia la desaparición de esos grados y a su sustitución por adverbios de cantidad o grado. Los superlativos se refuerzan a veces con adverbios de cantidad, por ejemplo, praeualidi nimis (Is. 31,I), grauissima ualde (I Sam. 5, I2), pessima uehementer (Ier. I4,I7); los positivos se refuerzan con dos adverbios de cantidad, por ejemplo, decora uehementer nimis (Ez. $\mathrm{I6}, \mathrm{I} 3)$, magna nimis ualde (Ez. 9,9), grandis nimis ualde (Ez. 37, I0), y hasta el comparativo se refuerza a veces con estos adverbios, por ejemplo, potentior ualde (Gen. 26,16). La primera parte de nuestro estudio puso claramente de relieve este aspecto del superlativo en el latín bíblico.

2. Algunos casos de sustitución del positivo por el superlativo representan una novedad absoluta en latín, como, por ejemplo, el modelo constituido por un positivo y una construcción preposicional (magnus e fratribus). La dependencia del hebreo de este tipo sintáctico no puede ponerse en duda. También se debe a influjo hebreo la frecuencia con que aparece el tipo de superlativo consistente en un adjetivo en grado positivo seguido de un genitivo partitivo (fortes illius).

3. Respecto al superlativo absoluto hebreo, expresado por la repetición del mismo nombre en plural (rex regum), hay que decir que en el latín bíblico su sentido es el de un verdadero superlativo, ya que no es más que la traducción literal de un modelo hebreo de superlativo. Essa misma fórmula en el latín profano podrá tener otro significado y podrá catalogarse ese genitivo como un genitivo partitivo, como hacen las gramáticas latinas ${ }^{1}$; pero en una gramática de latín bíblico habría que situarlo en otro contexto.

4. Con respecto al uso de adverbios de cantidad para expresar una idea superlativa, cabe señalar lo siguiente:

a) Ên el latín bíblico, nimis no está muchas veces por ualde ${ }^{2}$, sino que son dos adverbios perfectamente intercambiables, ya que ambos son o intentan ser la traducción de una misma palabra hebrea, mě'od. De hecho, nimis corresponde a esa palabra ro3 veces, y ualde, 70 veces. Por otra parte, nimis aparece en la Vulgata I44 veces, y ualde, I59 veces. Los traductores latinos de la Biblia los usan indistintamente, como puede comprobarse con toda claridad cuando traducen frases

1 Así Leumann-Hofmann-Szantyr, o. c., ibidem; M. Bassols, o. c., p. 86; A. Ernout-F. Thomas, o. c., p. 50 .

2 Como afirman Leumann-Hofmann-Szantyr, o. c., p. 163, § 97 a. 
totalmente iguales usando unas veces nimis y otras ualde. Algo parecido puede decirse de uehementer. También este adverbio significa para los traductores de la Biblia lo mismo que nimis y ualde. Las razones son las mismas. Vehementer corresponde a mĕ'od 28 veces, y en textos donde aparecen nimis y ualde podía haberse usado igualmente uehementer. Razones de pura preferencia son las que determinan el uso de uno en vez de otro de esos tres adverbios, y esto es válido, tanto si se trata de acompañar a adjetivos o a verbos. Satis, en cambio, es mucho menos usado. Sólo 6 veces corresponde a mĕod. Y su número total -20 veces-- es mucho menor que el de uehementer - 54 veces- y no tiene comparación con el de nimis - r44 veces - ni con el de ualde --I59 veces-.

b) Nimis se pospone siempre a la palabra que acompaña, menos 6 veces, -no 2 veces, como dicen Leumann-Hofmann-Szantyr ${ }^{1}$-, que son: Sal. 9I(92),6; Sal. 96(97),9; Sal. I38(r39),I7 (dos veces); Eccli2I,5; Eccli. I7,23. Y sin contar las 2 veces en que se usa la fórmula acu. mulativa nimis ualde (Ez. 9,9; 37,ro), en que nimis va delante, cuando podía haber ido igualmente detrás, dado que se trata de traducir el adverbio hebreo repetido mĕod mĕod. En la otra fórmula acumulativa uehementer nimis (Gen. I7,2; Ez. I6,13), que tiene el mismo origen -es decir, la traducción de mëod mĕod-, el traductor latino puso el nimis detrás, cuando pudo haberlo puesto delante, por la misma razón.

c) Tampoco es muy exacto afirmar, como hacen Leumann-Hofmann-Szantyr ${ }^{2}$, que ualde en la Vulgata "generalmente" se pospone a la palabra que acompaña. La realidad es que, de un total de I59 textos, va delante de la palabra 25 veces y 134 veces detrás.

d) Vehementer se pospone a la palabra 39 veces y se antepone I5. Satis, en cambio, se antepone a la palabra I4 veces y se pospone 6 .

e) Como habrá podido observarse por las estadísticas anteriores, los adverbios nimis, ualde y uehementer suelen ir detrás de la palabra que determinan. Este orden, por lo que respecta a la Biblia latina, depende fundamentalmente, $y$, casi podríamos decir, exclusivamente del orden de palabras en hebreo y griego, pues tanto mĕ od como $\sigma \phi \delta \delta \delta \alpha$ suelen ir casi siempre detrás de la palabra que determinan. Los traductores latinos raras veces han cambiado este orden. Por lo que respecta a satis, puede afirmarse lo mismo. Cuando traduce a mĕod siempre va detrás de la palabra. Cuando no tiene correspondencia con el hebreo, el traductor latino lo pone siempre delante. Cuando se trata de textos

1 O. c., p. 410, § 215 F; sólo citan Sal. 9I,6 y Eccli. 2I,5.

a c., p. $4 \mathrm{IO}, \S 2 \mathrm{I}_{5} \mathrm{~F}$. 
traducidos del griego (8 veces), va siempre delante de la palabra, coincidiendo este orden 4 veces con el griego.

f) Una breve comparación con el uso de estos mismos adverbios en Oribasio puede resultar interesante. En Oribasio, nimis con adjetivos suele ir delante de la palabra; en la Biblia latina, detrás. En Oribasio, nimis con verbos suele ir detrás de la palabra; en la Biblia latina también. Valde, en Oribasio, en contra del uso griego, siempre va delante de la palabra; en la Biblia latina va I34 veces detrás y sólo 25 delante. En Oribasio, uehementer y satis generalmente se posponen; en la Biblia latina, uehementer se pospone 39 veces y se antepone I5; mientras que satis generalmente se antepone ( 14 veces contra 6$)^{1}$. Estas diferencias tienen su última explicación en que el orden de palabras en la Biblia depende del texto hebreo, como dijimos antes, y los propios textos griegos o fueron traducidos del hebreo o escritos por autores hebreos.

g) El texto de Ier. 24,3 presenta un caso curioso de dos superlativos expresados por un positivo (ficus bonas) y otro positivo con ualde (ualde bonas), pero que, si no se atiende bien a la puntuación, dan la impresión de ser dos positivos para expresar un superlativo ${ }^{2}$. El texto completo dice así: Quid tu uides, Ieremia? Et dixi: Ficus, ficus bonas (= optimas), bonas ualde; et malas (= pessimas), malas ualde. Traducida la frase al lenguaje actual, y, teniendo presente la gradación que se siente hoy entre las formas de superlativo, expresadas por muy e -isimo, tendríamos la siguiente versión: ' $Y$ dije: (Veo) higos, higos muy buenos, bonísimos, y muy malos, malísimos' ${ }^{3}$.

En definitiva, la expresión del superlativo en la Biblia latina está muy influenciada por el hebreo.

Oliggario García de i.A Fuente

1 Cf. H. Morland, Die lat. Oribas.-Uebersetzungen, Oslo, 1932, p. 162; G. Hunger, Die Syntax des spätlateinischen Oribasius, Breslau, 1933, p. 25 ss. Los datos sobre Oribasio pueden verse en estos autores; los recogen también LeumannHofmann-Szantyr, o. c., ibidem.

2 Así los consideran Leumann-Hofmann-Szantyr, o. c., p. 163, § 97 a ("mit hebraisierend geminierten Positiv statt Superlativn).

3 Puede verse la versión que hemos dado nosotros mismos de este texto en Biblia, Barcelona, Herder, 1976, p. 817, traducción no tan literal como la que damos arriba, pero que da suficiente razón de los dos adjetivos '¿Qué ves, Jeremias?: Higos - respondi-. Los higos buenos son muy buenos; pero los malos, muy malos, que de tan malos no se pueden comer'. 